\title{
Football (soccer)-related spinal cord injury-reported cases from 1976 to 2020
}

\author{
Manoj K. Poudel $\mathbb{1}^{1} \cdot$ Andrew L. Sherman ${ }^{2}$ \\ Received: 10 August 2020 / Revised: 7 September 2020 / Accepted: 26 September 2020 \\ (c) The Author(s), under exclusive licence to International Spinal Cord Society 2020
}

\begin{abstract}
Study design An analysis of reported cases.

Objectives To analyze the existing data on soccer (international football)-related spinal cord injury (SCI).

Setting Cases of soccer (international football)-related SCI that were reported globally.

Methods PubMed/MEDLINE, EMBASE, and online news publication databases were searched.

Results Fourteen cases of football-related SCI that occurred between 1976 and 2020 were found. Average age at the time of injury was 19 and $86 \%$ of individuals were males. Eight of 14 individuals had vertebral fracture/dislocation, whereas two individuals had concomitant traumatic brain injury. Neurologically, 54\% had tetraplegia, 39\% had paraplegia, and $8 \%$ each suffered from hemiplegia and sensory deficit. Two cases could regain ability to walk with orthosis and four had full mobility with "Return to Play" (RTP). The mortality was $14 \%$.

Conclusions Younger males were most commonly affected. The most common etiology, vertebral level of injury, and neurological manifestation was fall, cervical spine, and tetraplegia respectively. More than $50 \%$ of the individuals with football-related SCI were able to walk or RTP after rehabilitation. Further studies are required to establish universal RTP criteria and formulate preventive measures.
\end{abstract}

\section{Introduction}

Spinal cord injury (SCI) is defined as a reversible or irreversible damage to the spinal cord due to trauma, disease, or degeneration. Individuals with SCI have complex medical and rehabilitation needs, and recovery may occur quickly or take months to years. Thus, SCI results in immense short and long-term costs for the individual, their family and health care systems.

In 2013, the World Health Organization and International Spinal Cord Society estimated that 250,000-500,000 people sustain SCI annually and the etiology in nearly $90 \%$ of the cases was trauma [1]. Data showed the most common cause of the traumatic SCI was transport/motor vehicle

Manoj K. Poudel

mannu.rooney@gmail.com

$\triangle$ Andrew L. Sherman

asherman@miami.edu

1 University of Miami/Jackson Health System, Miami, FL, USA

2 University of Miami Miller School of Medicine, Miami, FL, USA accidents, followed by falls (the most common cause after 60 year of age), violence/assault, and sports respectively $[1,2]$.

The highest prevalence of sports-related SCI is under 30 years of age. However, in the pediatric population the risk increases with age $[1,3]$. Regardless of etiology, males are more commonly affected than females [1, 2, 4]. Cervical injuries are most common in all sports-related SCIs and a significant number of sports-related SCIs can have associated bony injuries like fracture and dislocation of the spine $[4,5]$. Multiple studies have indicated tetraplegia is the most common degree of sports-related SCI; however, the ratio of tetraplegia to paraplegia and of incomplete to complete injury varied from one study to another $[6,7]$. Concomitant traumatic brain injury (TBI) is also reported in athletes with traumatic SCI and sports-related SCI can be disabling or fatal [8-11]. It can go undiagnosed because of minor or transient symptoms, injury without bony fracture or dislocation, concussion or subconcussive state $[12,13]$.

We wanted to evaluate the characteristics of soccer or international football-related SCI because as a relatively rare sport causing SCI, we were unable to find this information. Our objective is to describe current cases in the 
literature with regards to football-related SCI. This information is needed to formulate prevention strategies and help raise awareness among football players, team officials, and the general population.

\section{Methods}

An extensive search in PubMed/MEDLINE, EMBASE, and online news publications with the help of google search engine was done by using the phrases, "spinal cord injury soccer", "SCI soccer", "spinal cord injury football", and "SCI football". Twenty-three cases of football-related SCI were found. Sufficient data were not available for nine cases; therefore, they were excluded from review. Available data from the remaining 14 cases (seven from scientific journals and seven from online news publications) were collected and analyzed.

\section{Results}

Fourteen cases of SCI sustained while playing football were identified (Table 1). Cases were reported between 1976 and 2020. The greatest number were from Europe and North America with $79 \%$ of cases from developed countries. The male to female ratio was $6: 1$. Mean age at the time of injury was 19. There were no reported cases of football-related SCI under 8 years and above 35 years of age.

Most injuries were to the cervical region (71\%). The mechanisms of injury of the cervical SCI injuries were falls resulting in landing with the head on the ground in three cases (two fell after getting tackled by another player and one while performing goal celebration somersaults), collision in three cases, heading the ball in three cases (one was attributed to repetitive heading of the ball over a period of time), and hit by a falling goalpost in one case. One thoracic SCI occurred due to a fall on his upper back while stretching to intercept the ball and the other occurred due to hyperextension injury, again as a result of a fall. The lumbar SCI occurred in the player hit by the falling goalpost. The sacral SCI was due to a fall on lower back after collision with another player.

Out of 14 cases, 13 were diagnosed as acute SCI (93\%) at presentation. Out of these 13 cases, 12 suffered immediate symptoms, whereas the last player with sacral SCI developed symptoms $20 \mathrm{~min}$ after returning to play. One teenage male presented with progressive hypoesthesia of his extremities attributed to repeated heading of the ball over a period of time.

Eleven cases of the football-related SCI reported bony or ligamentous radiological findings, $43 \%$ had vertebral fracture/dislocation - five cases had fracture of spine, and one had subluxation of vertebrae. Two (14\%) of cases had concomitant TBI. Other associated injuries could not be analyzed because of insufficient data.

Total length of stay including acute rehabilitation was reported for four cases who spent (i) 34 days, (ii) 42 days, (iii) 11 months, and (iv) 18 months in the hospital. Of 13 survivors, seven sustained tetraplegia (54\%), four paraplegia (31\%), one hemiplegia $(8 \%)$, and one did not have a level of injury described due to only sustaining a sensory deficit $(8 \%)$.

Outcomes varied based on injury severity. Of 11 individuals with available data, four $(36 \%)$ were able to return to play (RTP). Average age of these individuals was 17.5 years; all were from developed countries, three had sustained minor trauma and none had bony injuries.

In the remaining cases, two (18\%) were able to walk with orthosis-average age was 13.5 years, both were females, both were from developed countries, both had spine fractures and one underwent surgical intervention. Five cases (45\%) were wheelchair dependent. The average age was 20.6 years, all were males, $80 \%$ were from the developed countries, $80 \%$ had fracture/dislocation of spine, tetraplegia to paraplegia ratio was $4: 1$, and $60 \%$ had surgery in the wheelchair dependent group.

One individual died during his acute SCI hospitalization while another died 2 years post injury. While there is no clear published cause of death in the first player, the latter died of respiratory causes.

\section{Discussion}

As of 2007, it was reported that 265 million people around the world played football [14]. Despite millions of players, reported cases of SCI are extremely low. Moreover, the regional distribution is uneven. This could be due to underreporting as only North America, Western Europe, Australia, and some other developed countries have a national reporting mechanism for traumatic SCI $[1,15]$. The other reason could be underdiagnosis which is possible with immediate death, minor or transient symptoms and when there is absence of bony injury [13]. Our analysis showed football-related SCI affects males more commonly than females, similar to other studies of sports-related SCI (except horse-riding), traumatic SCI, and overall SCI $[1,2,4]$. This could be because of higher proportions of males in the sport-related activities [16]. Sports-related SCI is also more common in younger populations due to the higher participation of this age group in the sporting activities (it is the second most common cause of SCI in populations younger than 15 years of age and third most common in populations between 16 and 30 years of age), and in pediatric populations the risk of the sports-related 


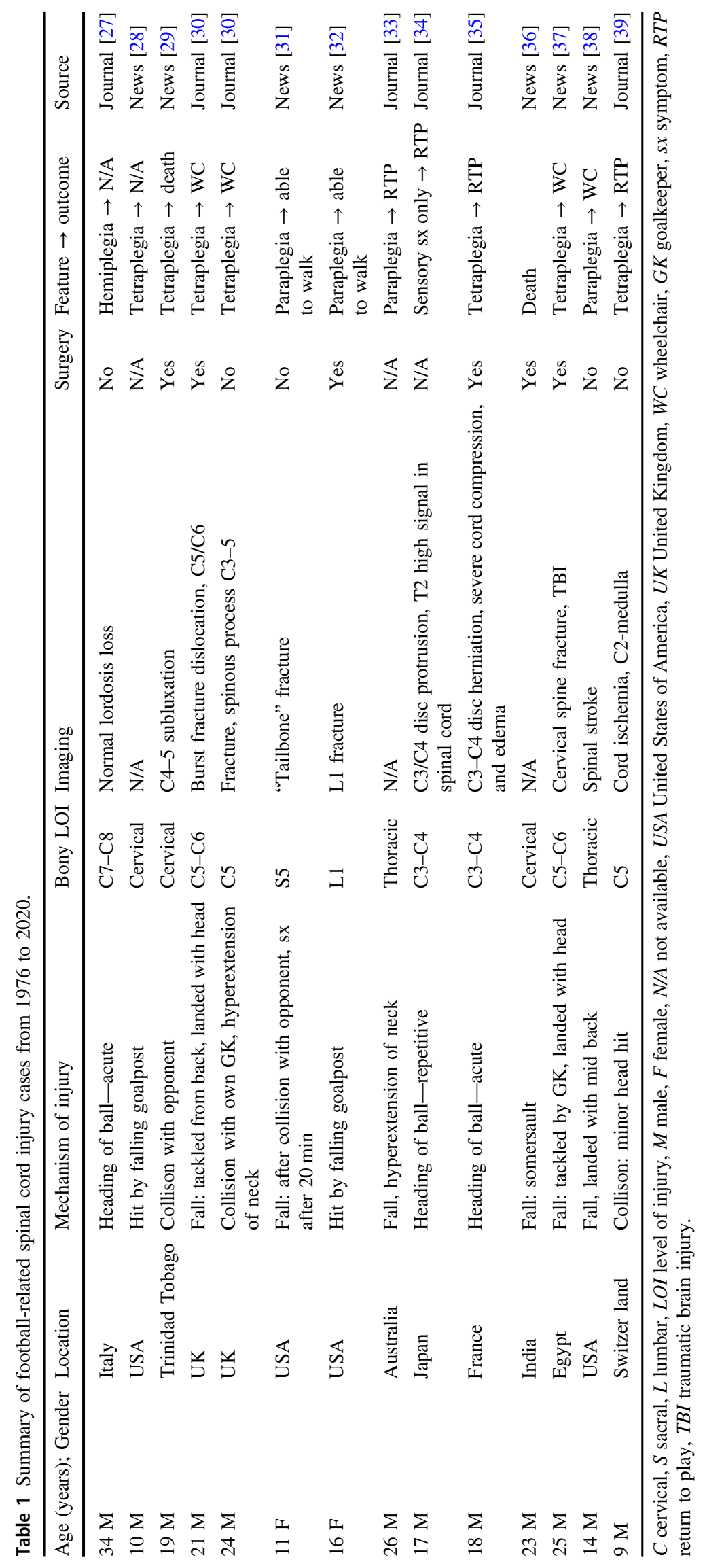


SCI increases with age for sports-related SCI, traumatic SCI and overall SCI [1-4]. Our series showed a similar pattern as the average age was 19 years and the incidence of football-related SCI increased with age in the pediatric population.

The etiology and mechanism of the sports-related SCI may vary across different sports; however, some contact sports, sports requiring running/kicking/twisting/jumping movements and celebration injuries may share similar mechanisms of injury. In our case series, the most common causes of football-related SCI were fall on the ground on head, collision, heading the ball, and getting hit by a falling goalpost. The latter two are not commonly encountered in other sports due to the nature of the sport and equipment used. Falling to the ground on the head and collision (with another individual or objects) are common in both contact and noncontact sports. An axial load on the spine (for all the spine levels) or compressive force to the top of the head (for cervical spine) are the most common mechanisms of sportsrelated SCI $[17,18]$. Individuals with congenital or acquired pathology of the spinal column such as canal stenosis, prior herniated disc, ligamentum flavum hypertrophy, etc. are at higher risk of sports-related SCI from participating in contact sports [18]. On top of the common etiologies/ mechanisms of injury, health care workers need to be aware that the football-related SCI can be caused by a single injury, even by a minor injury.

In football, head, neck, and trunk injuries which can cause SCI are much less common than injuries to the lower extremity [19]. Our review revealed a predominant number of cervical injuries $(71 \%)$ in football. However, footballrelated SCI is much less common than SCI related to diving, American football, gymnastics, baseball, basketball, hockey and skiing $[4,5]$. One study of 548 cases showed that $41 \%$ of sports-related SCI had bony injury of spine which is similar to the $43 \%$ in this series [4]. In addition to acute and obvious clinical manifestations, health care workers need to be aware that onset of symptoms can be delayed, transient, or progressive.

Management of football-related SCI starts from the field and includes proper evaluation of the player, as well as immobilization and transportation of the player to a definitive treatment center. Initial imaging modalities like X-ray and CT scan can miss a significant number of SCIs, especially in cases with cervical injury and in the pediatric population; hence, an MRI should be ordered at the least level of clinical suspicion [20]. In this small series an equal number of individuals with football-related SCI underwent conservative and surgical management. Determination of a treatment plan (conservative vs. surgical decompression) is based on the neurological findings, radiological features and the individual decision of the operative team [21, 22].
Multidisciplinary rehabilitation remains an essential part of the management strategy [23].

People with sports-related SCIs have been found to have higher physical independence, mobility, occupation, and community/social integration as compared to the nonathlete with SCI [24]. In this series, more than half of the athletes with football-related SCI were either able to walk again or RTP. There are no universally accepted guidelines for RTP for sports-related SCI. Minimum basic requirements are to be without pain, neurologically intact, and possessing full range of motion [25].

Many sports-related SCIs are felt to be preventable [1]. The authors believe that the screening strategies for the high-risk groups could be beneficial. Changing the rules for example, stopping the game immediately to seek medical attention after a suspected spine injury similar to the way it is done for suspected brain injury on the field, may help in early recognition and reduction of further damage of the spine/spinal cord. Development of age-specific precautions could be beneficial as a child's spine and supporting structures around it are not fully developed until a certain age. Special precautions taken during a tackle and avoiding risky goal celebration methods will also reduce the number of injuries. Awareness should be raised in the general population and the players, coaches, supporting staff and referees should be educated on football-related SCI that can occur during play. Early identification of cases with minor symptoms, short-lived symptoms and SCI without positive findings in x-rays/CT scans remains an important strategy $[12,13]$. The authors also believe that there should be standard RTP guidelines for all types of injuries to the spine or spinal cord. More studies are required to formulate detailed prevention strategies of football-related SCI because evidence-based prevention will lead to more reliable guidelines to prevent football-related SCI [26].

This is a small case series of reports of the scientific and gray literature. Thus, no conclusions can be drawn from the data. There was not uniformity in reporting the characteristics of SCI and the cases do not appear to adequately represent football-related SCI in developing countries where football continues to be extremely popular, as most cases included were reported from the developed countries. This series could not document the severity of the footballrelated SCI as there is no standardized data. Details on management during the acute hospital stay, rehabilitation and follow up visits were also not available. Thus, details on the need for mechanical ventilation, therapy strategies, length of stay in acute rehabilitation hospitals, discharge facilities, and post-injury complications could not be discussed. We are also unable to consider the financial cost of management of immediate and long-term needs of individuals with football-related SCI. Moreover, one of the 
important aspects of outcomes of SCI is the quality of life and mental health and there was no data on these issues.

\section{Conclusions}

This is the first case series that summarizes the patterns of football-related SCI. Even though the football-related SCI is rare, the number of cases is likely underreported. This is another reason that all countries should formulate and implement a national SCI reporting system. Further studies are required for players, health care workers, and sportsrelated personnel to better understand the prevention, patterns, management, outcomes, and to establish universal RTP criteria of football-related SCI.

\section{Compliance with ethical standards}

Conflict of interest The authors declare that they have no conflict of interest.

Publisher's note Springer Nature remains neutral with regard to jurisdictional claims in published maps and institutional affiliations.

\section{References}

1. International Perspectives on Spinal Cord Injury. World Health Organization and The International Spinal Cord Society. 2013. https://www.iscos.org.uk/sitefiles/WHO\%20international\% 20perspectives\%20on\%20SCI.pdf.

2. National Spinal Cord Injury Statistical Center, Facts and Figures at a Glance. Birmingham, AL: University of Alabama at Birmingham; 2020. https://www.nscisc.uab.edu/Public/Facts\%20and \%20Figures\%202020.pdf.

3. Parent S, Mac-Thiong JM, Roy-Beaudry M, Sosa JF, Labelle H. Spinal cord injury in the pediatric population: a systematic review of the literature. J Neurotrauma. 2011;28:1515-24.

4. Katoh S, Shingu H, Ikata T, Iwatsubo E. Sports-related spinal cord injury in Japan (from the nationwide spinal cord injury registry between 1990 and 1992). Spinal Cord. 1996;34:416-21.

5. Chan CW, Eng JJ, Tator CH, Krassioukov A. Spinal Cord Injury Research Evidence Team. Epidemiology of sport-related spinal cord injuries: a systematic review. J Spinal Cord Med. 2016;39:255-64.

6. Boran S, Lenehan B, Street J, McCormack D, Poynton A. A 10year review of sports-related spinal injuries. Ir $\mathrm{J}$ Med Sci. 2011;180:859-63.

7. Ye C, Sun T, Li J, Zhang F. Pattern of sports- and recreationrelated spinal cord injuries in Beijing. Spinal Cord. 2009;47:857-60.

8. Paiva WS, Oliveira AM, Andrade AF, Amorim RL, Lourenço LJ, Teixeira MJ. Spinal cord injury and its association with blunt head trauma. Int J Gen Med. 2011;4:613-5.

9. Kristen KL, Yau RK, Register-Mihalik J, Marshall SW, Thomas LC, Wolf S, et al. Traumatic brain and spinal cord fatalities among high school and college football players-United States, 2005-14. MMWR Morb Mortal Wkly Rep. 2017;65:1465-9.
10. Indian footballer dies after goal celebration ends in tragedy. Goal. com. 2014. https://www.goal.com/en/news/14/asia/2014/10/20/ 5295341/indian-footballer-dies-after-goal-celebration-ends-in.

11. Famous Career-Ending Sports Injuries. Healthgrades.com. 2018. https://www.healthgrades.com/right-care/sports-medicine/famouscareer-ending-sports-injuries.

12. Nagoshi N, Tetreault L, Nakashima H, Nouri A, Fehlings MG. Return to play in athletes with spinal cord concussion: a systematic literature review. Spine J. 2017;17:291-302.

13. Cirak B, Ziegfeld S, Knight VM, Chang D, Avellino AM, Paidas CN. Spinal injuries in children. J Pediatr Surg. 2004;39:607-12.

14. Big Count. FIFA. 2007. https://www.fifa.com/mm/document/fifa facts/bcoffsurv/emaga_9384_10704.pdf.

15. Lee BB, Cripps RA, Fitzharris M, Wing PC. The global map for traumatic spinal cord injury epidemiology: update 2011, global incidence rate. Spinal Cord. 2014;52:110-6.

16. Deaner RO, Smith BA. Sex differences in sports across 50 societies. Cross Cult Res. 2013;47:268-309.

17. Wilson JB, Zarzour R, Moorman CT 3rd. Spinal injuries in contact sports. Curr Sports Med Rep. 2006;5:50-5.

18. Bailes JE, Petschauer M, Guskiewicz KM, Marano G. Management of cervical spine injuries in athletes. J Athl Train. 2007;42:126-34

19. Pfirmann D, Herbst M, Ingelfinger P, Simon P, Tug S. Analysis of injury incidences in male professional adult and elite youth soccer players: a systematic review. J Athl Train. 2016;51:410-24.

20. Szwedowski D, Walecki J. Spinal cord injury without radiographic abnormality (SCIWORA)—clinical and radiological aspects. Pol J Radiol. 2014;79:461-4.

21. El Masri YWS. Traumatic spinal injury and spinal cord injury: point for active physiological conservative management as compared to surgical management. Spinal Cord Ser Cases. 2018;4:14.

22. Mourelo Fariña M, Salvador de la Barrera S, Montoto Marqués A, Ferreiro Velasco ME, Galeiras, Vázquez R. Update on traumatic acute spinal cord injury. Part 2. Med Intensiva. 2017;41:306-15.

23. Sabharwal S, Wierbicky J, Nesathurai S. Spinal cord injury: cervival, thoracic and lumbosacral. In: Frontera W, Silver J, Rizzo T editors. Essentials of physical medicine and rehabilitation. 2nd edn. Amsterdam: Elsevier; 2008. p. 859-85.

24. Hanson CS, Nabavi D, Yuen HK. The effect of sports on level of community integration as reported by persons with spinal cord injury. Am J Occup Ther. 2001;55:332-8.

25. Huang P, Anissipour A, McGee W, Lemak L. Return-to-play recommendations after cervical, thoracic, and lumbar spine injuries: a comprehensive review. Sports Health. 2015;8:19-25.

26. Bellon K, Kolakowsky-Hayner SA, Chen D, McDowell S, Bitterman B, Klaas SJ. Evidence-based practice in primary prevention of spinal cord injury. Top Spinal Cord Inj Rehabil. 2013;19:25-30.

27. Scoppetta C, Vaccario M. Cervical central cord syndrome after heading a football. Lancet. 1978;311:1269.

28. Soccer Goal Deaths and Injuries 1979-2017. Anchored for safety. 2017. http://anchoredforsafety.org/incidents.html.

29. Ex-Trinidad player Lee dies at 21. LA Times. 2003. https://www. latimes.com/archives/la-xpm-2003-mar-11-sp-soccer11-story. html.

30. Silva P, Vaidyanathan S, Kumar BN, Soni BM, Sett P. Two case reports of cervical spinal cord injury in football (soccer) players. Spinal Cord. 2005;44:383-5.

31. SCI Superstar: Mackenzie Saunders. Spinalpedia.com. 2019. https://spinalpedia.com/blog/2019/06/mackenzie-saunders.

32. Durango girl healing from serious spinal injury. Durango Herald. 2009. https://durangoherald.com/articles/3529.

33. Ojo OA, Kaye AH. An unusual cause of spinal cord compression. Images Neurosci. 2010;17:86. 
34. Kato Y, Koga M, Taguchi T. Chronic spinal cord injury in the cervical spine of a young soccer player. Orthopedics. 2010;33:357.

35. Diabira S, Morandi X. Post-traumatic herniated cervical disk. N Engl J Med. 2012;366:462.

36. Indian footballer dies from fall after back-flip goal celebration. ESPN.com. 2014. https://www.espn.com/soccer/league-name/ story/2098397/headline.

37. African Confederation Cup: Player recovering after spinal injuries. ESPN.co.uk. 2015. https://www.espn.co.uk/football/blog-footba 11-africa/story/2539482/african-confederation-cup-player-suffersspinal-injuries.

38. Soccer player, 14, recovering from spinal stroke. Chicago Tribune. 2015. https://www.chicagotribune.com/sdut-teenrecovering-from-spinal-stroke-at-soccer-game-2015jul29story.html.

39. Dünner C, Hackenberg A, Wille D, Cannizzaro V. Cervical spinal ischemia after minor head trauma. Swiss Medical Weekly. 2017;147:30. 